\title{
Maternal socio-economic factors and the risk of premature birth and low birth weight in Cyprus: a case-control study
}

\author{
Paraskevi Stylianou-Riga ${ }^{a}$ Panayiotis Kouis ${ }^{b}$ Paraskevi Kinni ${ }^{b}$ Angelos Rigas ${ }^{c}$ Thalia Papadouria \\ Panayiotis K. Yiallouros ${ }^{\mathrm{b}}$ Mamas Theodorou ${ }^{\mathrm{d}}$ \\ a Neonatal Intensive Care Unit, "Archbishop Makarios III" Hospital, Nicosia, Cyprus \\ b Medical School, University of Cyprus, Nicosia, Cyprus \\ c Apollonion Hospital, Nicosia, Cyprus \\ d Faculty of Economics and Management, Open University of Cyprus, Nicosia, Cyprus
}

\section{Background}

Prematurity and low birth weight are significant predictors of perinatal morbidity and mortality. Prematurity and low birth weight are influenced by the overall health and socioeconomic status of the pregnant mother. Cyprus is characterized by the highest premature birth rate in Europe $(13.1 \%$ based on 2014 data). In this study we investigated the association of maternal demographic, clinical, social and economic characteristics with premature delivery and low neonatal birth weight among premature infants. In addition we examined the effect of clusters of risk factors on prematurity risk.

\section{Methods}

\section{Study Population}

Our case-control study included 349 term controls and 348 preterm labour cases. The clinical data were obtained from hospital records and the socioeconomic data collected through a structured self-administered questionnaire including parameters such us basic demographics, income, education, working conditions, gestational diabetes, stress, and smoking.

\section{Statistical Analysis}

We used multivariable logistic regression to estimate odds ratio $(\mathrm{OR})$ and $95 \%$ confidence intervals $(\mathrm{Cl})$ of preterm delivery risk factors. Among cases, the relationship between different characteristics and gestational age as well as birth weight were examined using the Pearson correlation coefficient and adjusted analyses were performed using a multivariate linear regression model. The OR of combinations of risk factors with significant overlap was calculated using binary logistic regression.

Table 1. Association between maternal risk factors and prematurity

\begin{tabular}{llll}
\hline Risk Factor & Contrast & Odds Ratio $(95 \% \mathrm{Cl})$ & $P$-value \\
\hline Age at child birth & Continuous & $1.12(1.06,1.18)$ & $<0.001$ \\
Pre-gestation BMI & Continuous & $0.96(0.92,1.01)$ & 0.11 \\
Gestational diabetes & Categorical & $0.53(0.30,0.97)$ & 0.04 \\
Depression & Categorical & $1.38(0.25,7.61)$ & 0.71 \\
Stress & Categorical & $8.5(3.03,23.89)$ & $<0.001$ \\
Family status & Categorical & $1.11(0.57,2.15)$ & 0.77 \\
Manual labour & Categorical & $1.54(0.90,2.65)$ & 0.11 \\
Long working hours & Categorical & $3.77(2.08,6.84)$ & $<0.001$ \\
\hline
\end{tabular}

\section{Results}

Premature delivery was associated with greater maternal age (OR: 1.12, 95\% Cl: 1.06-1.18), absence of gestational diabetes (OR: $0.53,95 \% \mathrm{Cl}: 0.30-0.97$ ), long working hours (OR: 3.77, 95\% Cl: 2.08-6.84) and emotional stress (OR: 8.5, 95\% Cl: 3.03-23.89).Within the cases group, emotional stress was also associated with lower birth-weight ( $\beta$ : -323.68 (95\% $\mathrm{Cl}$ : -570.36, -77.00). The figure, presents the different combinations of significant maternal risk factors in a 3-way Venn diagram. The most frequent combination was advanced maternal age and long working hours (OR: 5.17, 95\% Cl: 2.6010.30) followed by the combination of advanced maternal age and stress (OR: 9.89, 95\% Cl:3.66-26.72).

Fig. 1 Overlap of significant risk factors for prematurity. Long Hours Stress

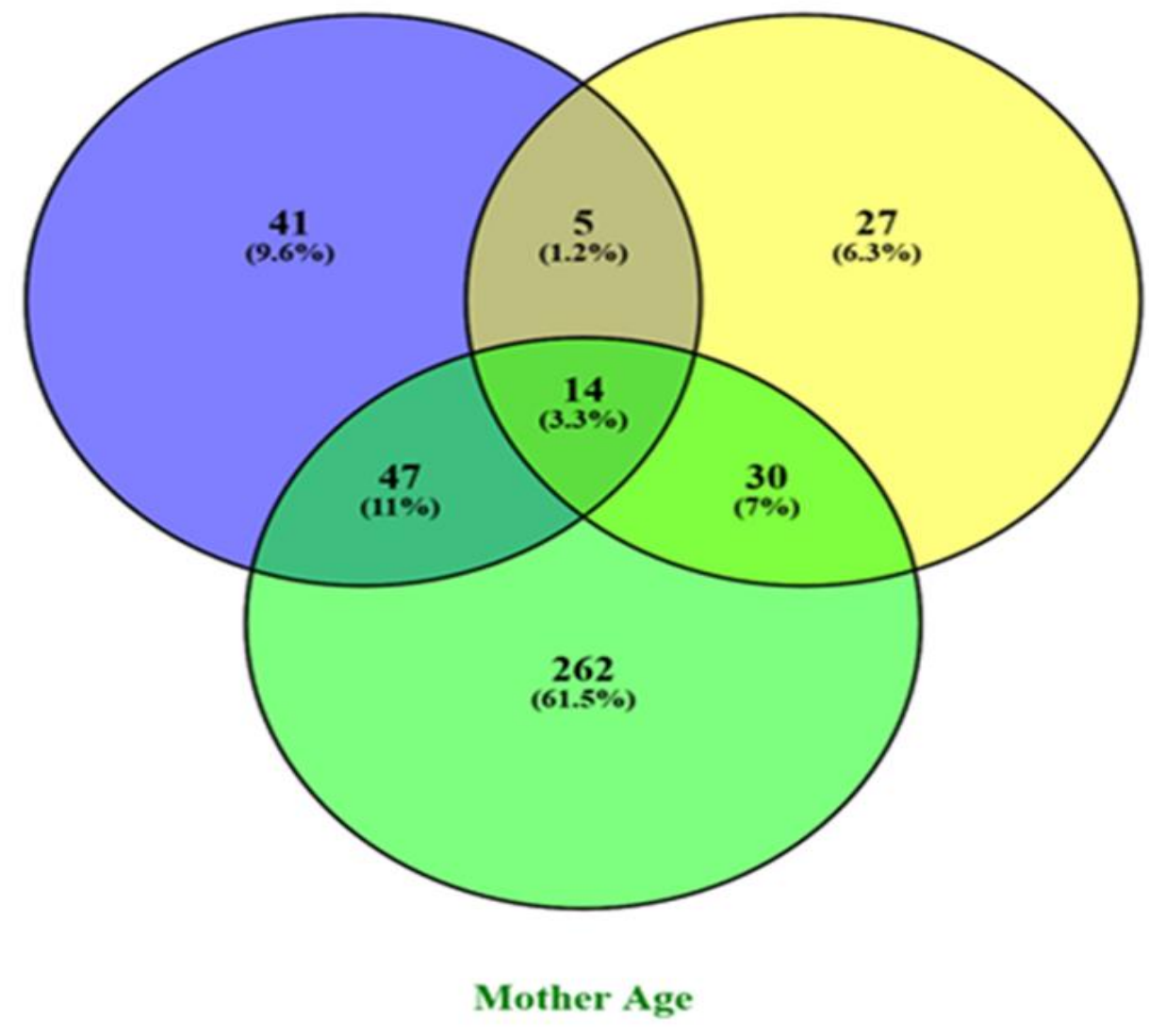

Table 2. Association between maternal risk factors and birth weight

\begin{tabular}{llll}
\hline Risk Factor & Contrast & Coefficient $(95 \% \mathrm{Cl})$ & $P$-value \\
\hline Age at child birth & Continuous & $-16.00(-33.52,1.54)$ & 0.07 \\
Pre-gestation BMI & Continuous & $13.37(-8.43,35.16)$ & 0.23 \\
Gestational diabetes & Categorical & $201.13(-69.37,471.64)$ & 0.14 \\
Depression & Categorical & $-34.81(-647.09,577.46)$ & 0.91 \\
Stress & Categorical & $-323.68(-570.36,-77.00)$ & 0.01 \\
Family status & Categorical & $145.16(-66.15,356.46)$ & 0.18 \\
Manual labour & Categorical & $-154.636(-362.05,52.77)$ & 0.14 \\
Long working hours & Categorical & $123.25(-80.72,327.22)$ & 0.24 \\
\hline
\end{tabular}

\section{Conclusions}

Stress, prolonged working hours and advanced maternal age at childbirth, increase the risk of prematurity and low birth weight in Cyprus. In addition, combination of adverse socio-economic risk factors has a cumulative effect on prematurity risk.

Dr Paraskevi Stylianou-Riga Neonatal Intensive Care Unit, "Archbishop Makarios III" Hospital; Nicosia, Cyprus Email: skeviriga@yahoo.com 